Supporting Information

\title{
Graphene monoxide bilayer as a high performance on/off switching media for nanoelectronics
}

\author{
Jungwook Woo, Kyung-Han Yun, Yong-Chae Chung* \\ Department of Materials Science and Engineering, Hanyang University, \\ 222 Wangsimni-ro, Seongdong-gu, Seoul, 04763, Republic of Korea \\ *Email address: yongchae@,hanyang.ac.kr
}

In the supporting Information, some additional results for better understanding of our study are included: The energies versus interlayer distance, differential charge densities for the same interlayer distance, the partial density of states (PDOS) of the GMO monolayer and bilayers, the planar-averaged differential charge density and electric field induced by charge redistribution, the band gaps as a function of electric field for the same interlayer distance, The band gap as a function of electric field using hybrid functionals and the band structure and PDOS of the GMO bilayers (the hol-O and hol-br).

Keyword: graphene monoxide, giant Stark effect, band gap, electric field, first-principles calculation 
The energies of each GMO bilayers as a function of interlayer distance
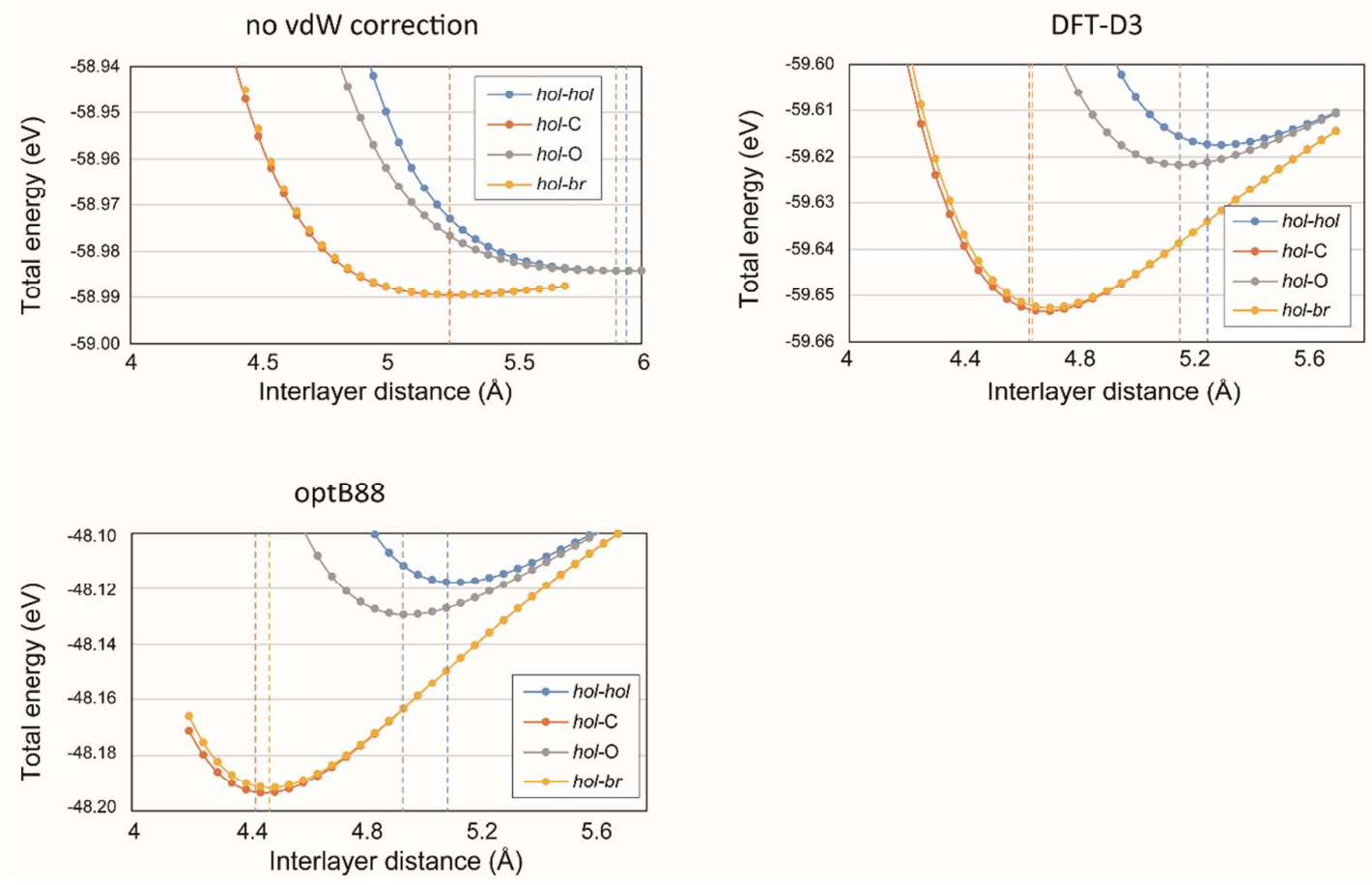

Figure S1. The calculated total energies of each systems versus interlayer distance with difference vdW corrections (without vdW correction (typical PBE calculation), DFT-D3 correction and optB88-vdW scheme). 
Differential charge density of the GMO bilayers for the same interlayer distance (5.24 $\AA)$

(a)

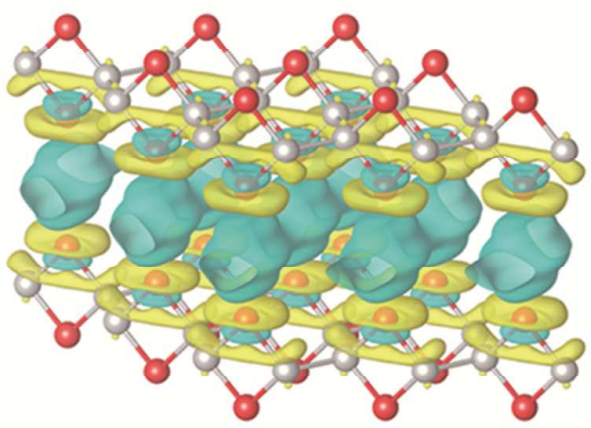

hol-hol

(c)

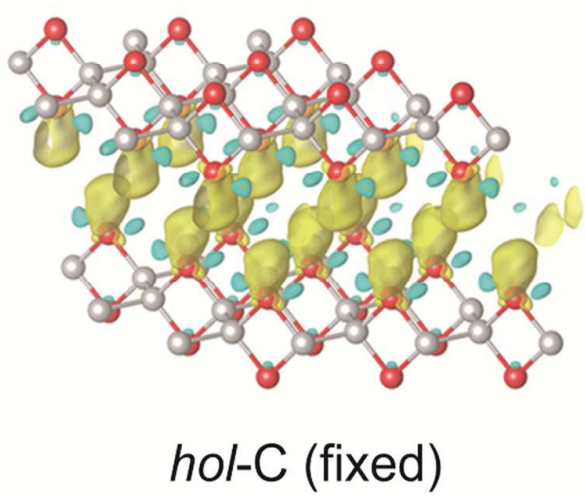

(b)

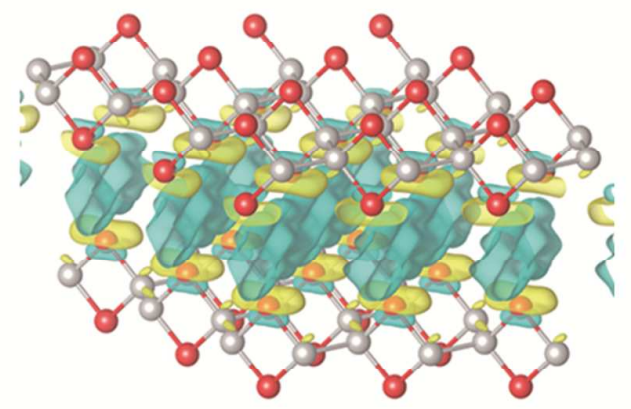

hol-O

(d)

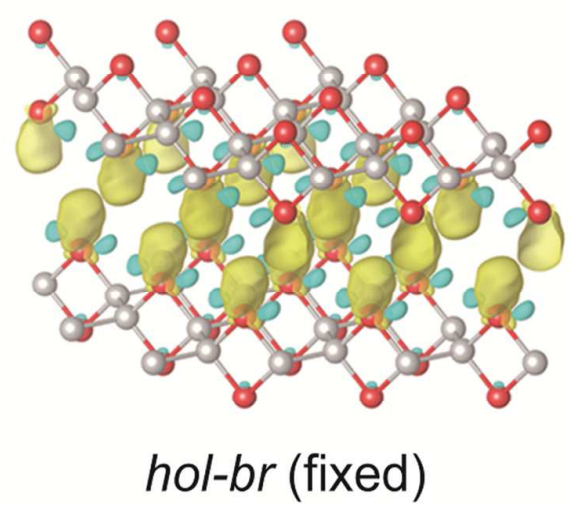

Figure S2. Differential charge density of the GMO bilayers for the same interlayer distance (5.24 $\AA$ ) ((a) the hol-hol, (b) hol-O, (c) hol-C and (d) hol-br) relative to the GMO monolayer. The yellow and green isosurfaces correspond to the accumulation and depletion region in the values of $0.0002 e / \AA^{3}$, respectively. The red and gray atoms represent the carbon and oxygen atoms, respectively. 
(a)

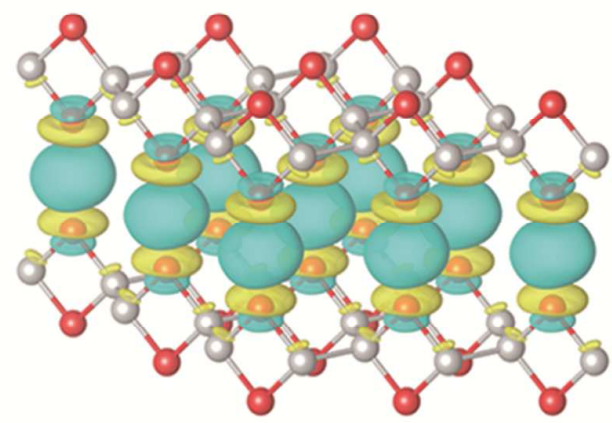

$$
\text { hol-hol (fixed) }
$$

(c)
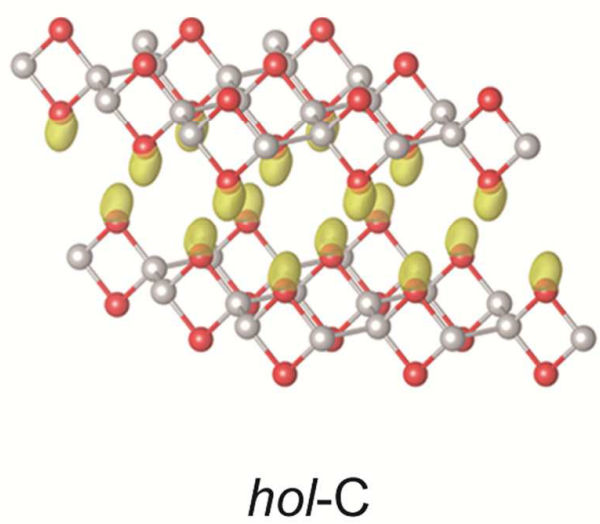

(b)

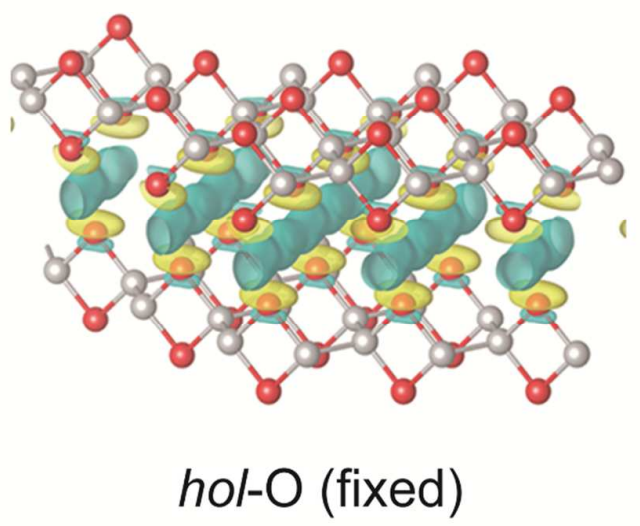

(d)

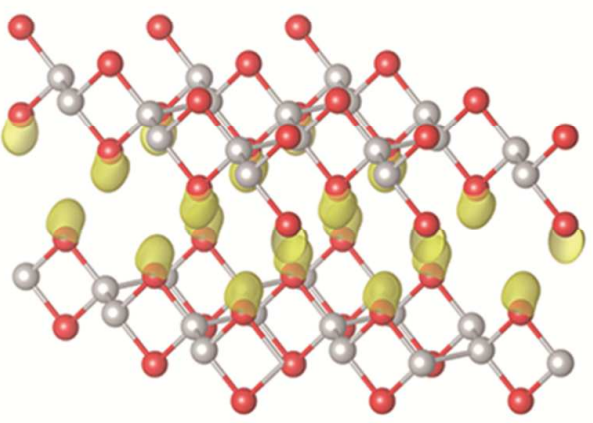

$h o l-b r$

Figure S3. Differential charge density of the GMO bilayers for the same interlayer distance (4.60 A) ((a) the hol-hol, (b) hol-O, (c) hol-C and (d) hol-br) relative to the GMO monolayer. The yellow and green isosurfaces correspond to the accumulation and depletion region in the values of $0.002 e / \AA^{3}$, respectively. The red and gray atoms represent the carbon and oxygen atoms, respectively. 
The partial density of states (PDOS) of the GMO monolayer and GMO bilayers

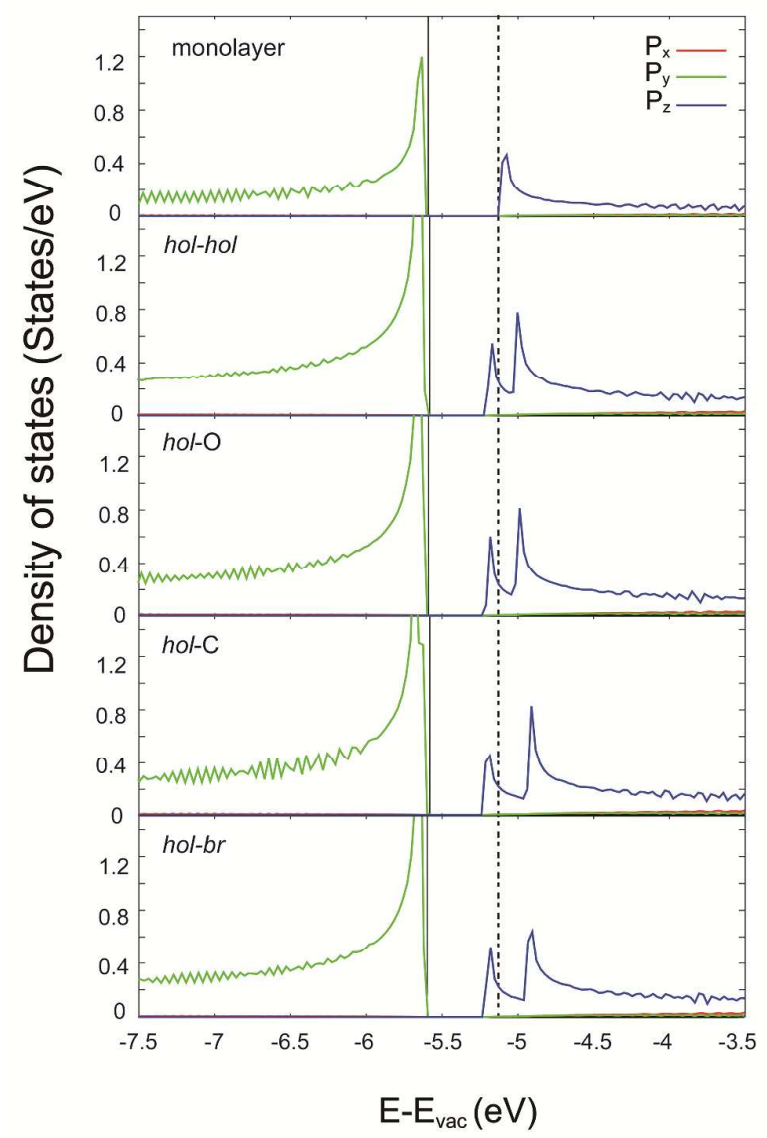

Figure S4. The partial density of states of the GMO monolayer and GMO bilayers (the holhol, hol-O, hol-C, and hol-br) without an electric field. The dashed lines and solid indicate the CBM energy level of the monolayer and the fermi level, respectively. 
The planar-averaged differential charge density of GMO bilayers with applying electric field
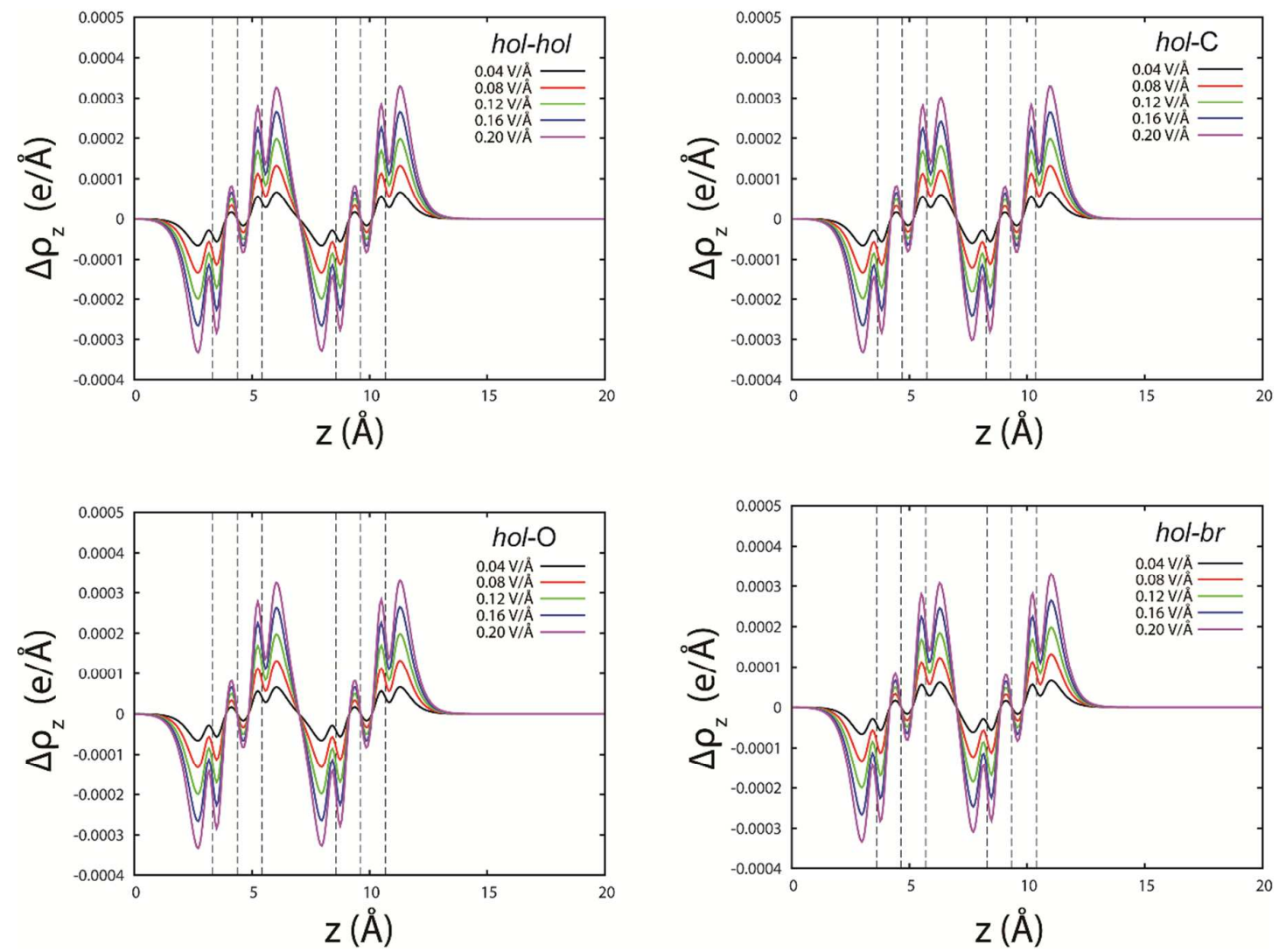

Figure S5. The planar-averaged differential charge density of GMO bilayers (hol-hol, hol-C, hol-O, hol-br) as a function of $\mathrm{z}$ coordinate with applying electric field. 
The electric field induced by the charge redistribution of GMO bilayers
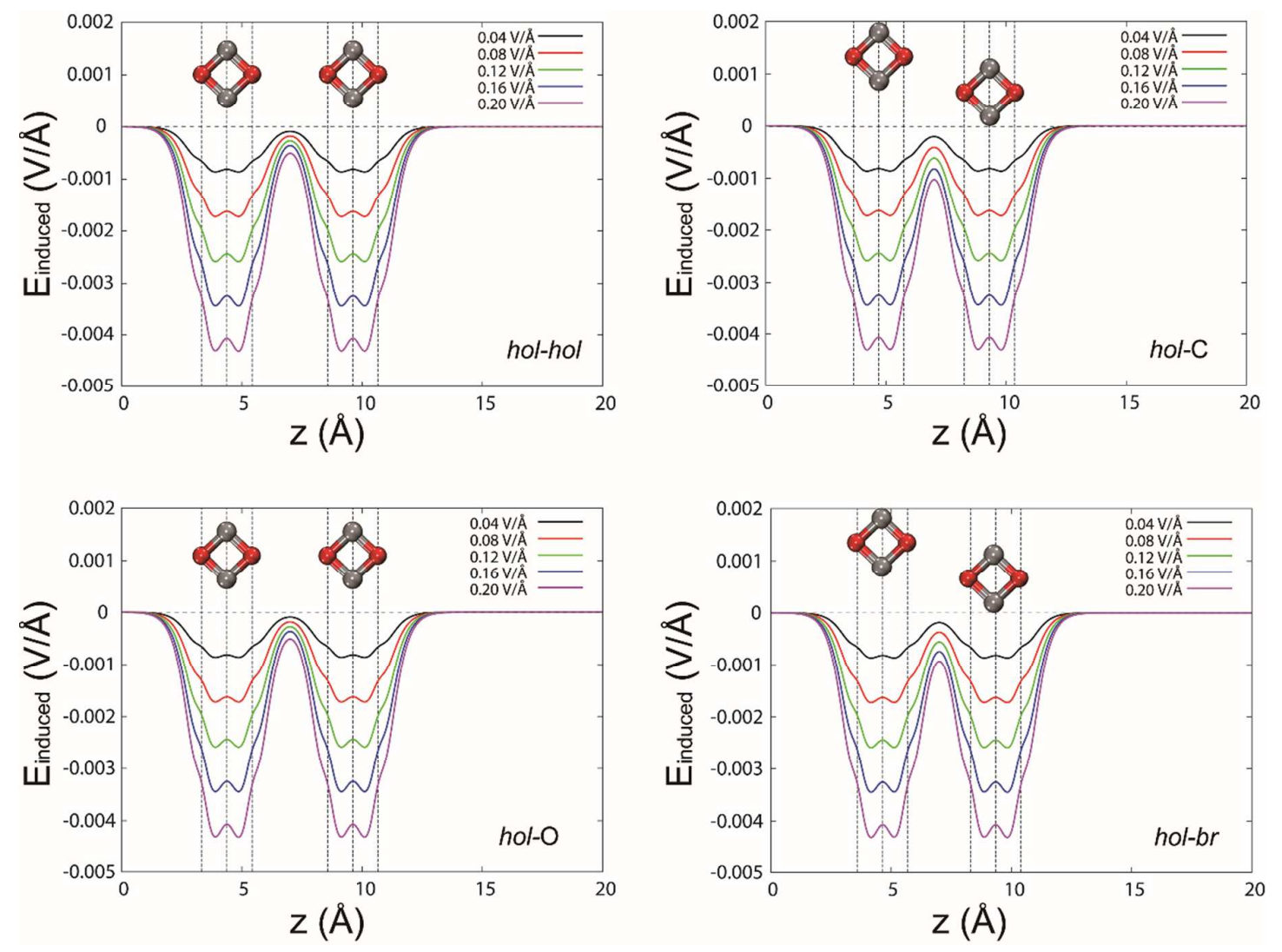

Figure S6. The electric field induced by the charge redistribution of GMO bilayers (hol-hol, hol-C, hol-O, hol-br). The electric field induced the charge redistribution is calculated by integrating the planar-averaged differential charge density (Figure S7). The charge redistribution in Figure S7 will cause the force on the atoms of GMO bilayers. However, all cases of resulting force by the charge redistribution are under the Hellmann-Feynman force on each atoms $(0.01 \mathrm{eV} / \AA ̊)$. 
The band gap as a function of the external electric field for the GMO bilayers with same interlayer distance

(a)

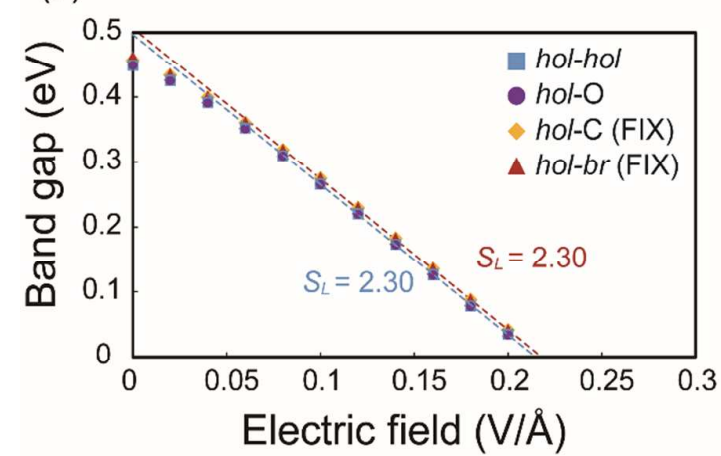

(b)

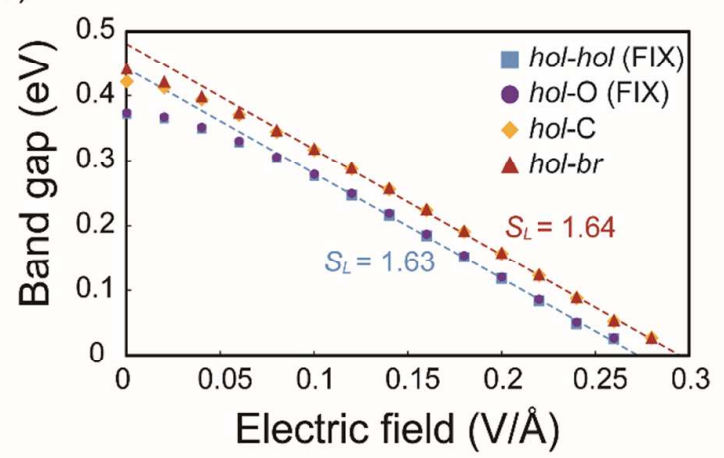

Figure S7. The band gap as a function of the external electric field for the GMO bilayers fixed at (a) the longer interlayer distance (5.24 $\AA$ ) and (b) the shorter interlayer distance (4.60^), respectively. The dashed lines are fitted to the linear part of the band gap curve. The magnitudes of the slopes $\left(S_{L}\right)$ of the linear band gap changes are indicated. 
The band gap as a function of electric field using hybrid functionals

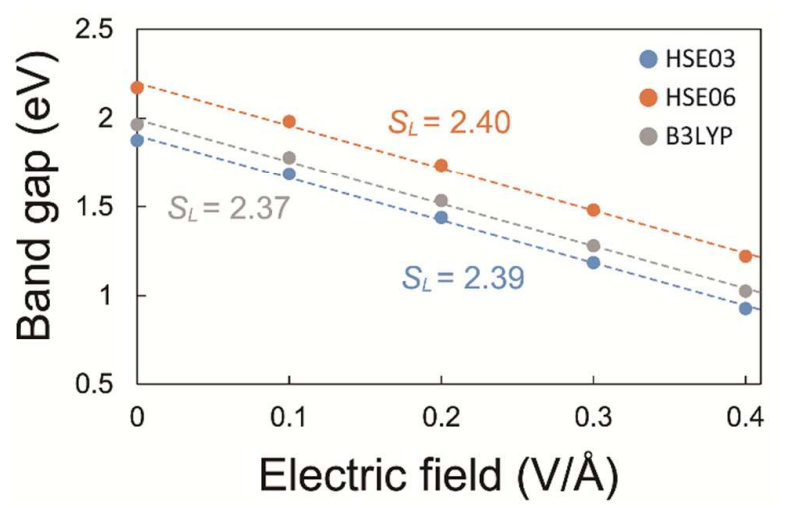

Figure S8. The band gap as a function of the external electric field using several hybrid functional (HSE03, HSE06 and B3LYP) for hol-hol structure as a representative of GMO bilayers. The dashed lines are fitted to the linear part of the band gap curve. The magnitudes of the slopes $\left(S_{L}\right)$ of the linear band gap changes are indicated. 
The band structure and PDOS of the GMO bilayers (the hol-O and hol-br)
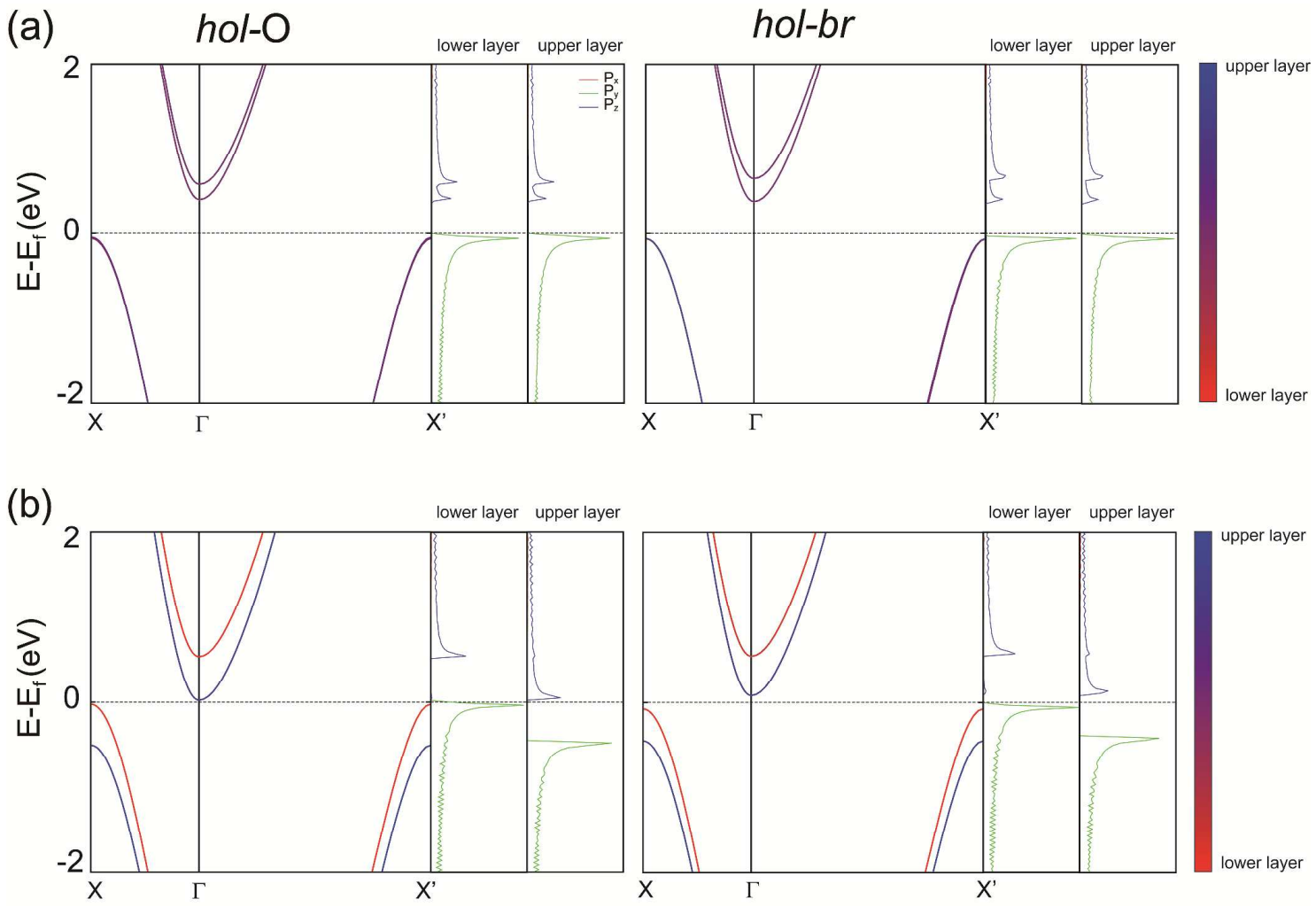

Figure S9. The band structures and the partial density of states (PDOS) (a) without and (b) with an electric field $(0.2 \mathrm{~V} / \AA)$ for the GMO bilayers (the hol-O and hol-br). The color bar indicates the degree of contributions of each band to the upper (blue) or lower (red) layers. 